Ann. Biol. anim. Bioch. Biophys., I964, 4 (3) 273-286.

\title{
LE TESTAGE DES TAUREAUX SUR LA PRODUCTION EN MATIĖRE AZOTÉE DU LAIT DE LEURS FILLES RÉSUlTATS PRÉliminaIREs ( $\left.{ }^{(}\right)$(Première Partie)
}

\author{
M. POUTOUS \\ Avec la collaboration technique de Suzanne Crochez \\ et Lucie TASSENCOURT \\ Station centrale de Génétique animale, \\ Centre national de Recherches zootechniques, Jouy-en-Josas (Seine-et-Oise)
}

\section{SOMMAIRE}

Une analyse a été faite sur I 584 premières lactations des filles de 45 taureaux du Centre de

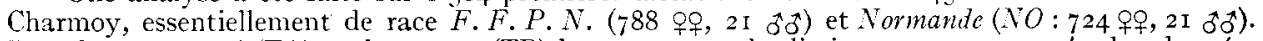
Pour le taux azoté (TA) ou butyreux (TB) la moyenne et les limites comprenant $2 / 3$ des données autour de la moyenne sont :

$$
\begin{array}{ll}
\text { F.F.P.N..TA }=3 \mathrm{~T}, \mathrm{O} ; 28,9-32,8 & \text { TB }=35,9 ; 33,0-38,6 \\
\text { NO .....TA }=33,4 ; 3 \mathrm{I}, \mathrm{I}-35,3 & \mathrm{~TB}=38,6 ; 35,3-4 \mathrm{I}, 9
\end{array}
$$

Les quantités totales, par lactation, de lait, de matières azotées et de matières grasses sont très variables $(C . V . \geqslant 30$ p. Ioo), très liées phénotypiquement (coefficient de corrélation $r \geqslant 0,95$ ) et génétiquement $(r \geqslant 0,90)$. Les coefficients d'héritabilité, un peu plus élevés pour la quantité de lait, sont voisins $\left(0,16 \leqslant h^{2} \leqslant 0,20\right)$. Leur variabilité phénotypique provient en partie de la durée de lactation $(r \geqslant 0,67)$. La variabilité des taux est nettement moindre (C.V.: TA $=6,4$; TB $=8,6)$, leurs héritabilités semblables avec de grandes différences entre les races $\left(F . F . P . N .: h^{2} \mathrm{TA}=0, \mathbf{1} 7\right.$; $h^{2} \mathrm{~TB}=0,10 . N O: h^{2} \mathrm{TA}=0,4^{2} ; h^{2} \mathrm{~TB}=0,37$ ). Les coefficients de corrélation phénotypiques et génétiques entre les taux valent : en race $F . F . P . N$. o,61 et 0,46 ; en race $N O 0,50$ et 0,63 ; en moyenne intra-race : 0,54 et 0,57 .

On a trouvé des corrélations phénotypiques peu élevées entre taux et durée de lactation (en moyenne : $r \mathrm{TA}=0,09 ; r \mathrm{~TB}=0, \mathrm{I} 4)$ et pratiquement nulles entre taux et quantité de lait $(r \not 0)$ sauf à durée de lactation constante $(F . F . P . N .: r \mathrm{TA} \# r$ TB $=-0, \mathrm{I} 6)$. La quantité de matières azotées dépend peu du taux azoté (en moyenne $r=0,19$ ) et moins encore du taux butyreux (en moyenne $r=-0,06$ ). Cette tendance se retrouve entre quantité de matière grasse et taux butyreux ou azoté (en moyenne $r \mathrm{~TB}=0,27, r \mathrm{TA}=0, \mathrm{I} 4$ ). Les liaisons génétiques entre quantité de matières azotées et taux azoté ou bien entre quantité de matières grasses et taux butyreux sont faibles (en moyenne $r \mathrm{TA} . \mathrm{MA}=0,02 ; r \mathrm{~TB} . \mathrm{MG}=0, \mathrm{Io}$ ). Les corrélations génétiques croisées sont négatives (en moyenne $r$ 'TA. $\mathrm{MG}=-0,07 ; r \mathrm{~TB} . \mathrm{MA}=-0,06$ ). Les corrélations génétiques négatives entre la quantité de lait et les taux sont plus intenses en race $N O(r$ TA $=-0,39$; $r \mathrm{~TB}=-0,35)$ qu'en race $F . F . P . N .\left(r \mathrm{TA}=-0,10 ; r \mathrm{~TB}=-0, \mathrm{I}_{4}\right)$.

L'écart-type génétique de la matière azotée est inférieur à celui de la matière grasse (MA/MG = I963).

(1) Un résumé de ce mémoire a été présenté aux Journées de l'A. L. F. (Nancy, juin 1963) (Poutous,

Annales de Biologie animale. - 1964 . 
o,84). Il en est de même pour le taux azoté par rapport au taux butyreux (TA/TB:F.F.P.N. =0,89 $N O=0,69)$. Par des corrélations partielles, à quantité de lait constante, entre quantité et taux, on a réestimé la corrélation phénotypique entre les taux à 0,57 , la corrélation génétique devant dépasser 0,67 . L'effet de la sélection sur ces résultats et l'utilité du contrôle azoté seront discutés dans une étude ultérieure.

\section{INTRODUCTION}

Les problèmes que posent la production et l'utilisation des constituants azotés du lait intéressent de plus en plus éleveurs et transformateurs. C'est ainsi qu'en I963, 1'Association Laitière Française a retenu ce sujet comme thème de ses Journées d'étıdes. Ceci s'explique par une meilleure connaisance qu'auparavant de la valeur nutritionnelle des éléments non gras du lait, principalement de celle des protéines. D'autre part, on attache plus d'importance à la matière azotée à cause des difficultés actuelles du marché, car l'écoulement semble plus facile pour le fromage que pour le beurre. Il est donc normal qu'on cherche à produire des laits riches en constituants azotés, matière première indispensable à la fabrication des fromages.

Plusieurs mises au point sur les connaissances acquises dans ce domaine ont été publiées par Robertson et al. (I956), Tyler (I958), Legates (I960), et JohansSON (I96I). Les plus récentes sont celles d'AURIoL (I962), et d'AURIoL et JARriGe (I962). Les auteurs de ces revues, après étude critique de la documentation bibliographique, s'accordent à admettre que le taux azoté ${ }^{(1)}$ du lait est surtout affecté par la sous-nutrition énergétique. Il semble par contre difficile et, en tout cas, non économique de vouloir l'améliorer par une suralimentation des animaux au-delà des normes usuelles. Pour reprendre en partie les conclusions d'AUrior et JARrige, l'animal n'atteint son niveau maximum de production qu'en milieu favorable, mais ce plafond, seule la sélection peut utilement 1e déplacer.

Or il semble possible de pratiquer une telle sélection puisqu'il existe des différences génétiques entre races et même entre individus d'une même race. Le coefficient d'héritabilité du taux de protéines serait relativement élevé et voisin de celui du taux butyreux. Ces deux caractères seraient, de plus, liés par des corrélations phénotypique et génétique positives. Par contre, les opinions divergent sur l'intérêt d'une sélection sur le rapport $\frac{\text { taux azoté }}{\text { taux butyreux }}$. Préconisée par AURIoL et JARRIGE, son efficacité pratique serait limitée, selon Johansson, par les faibles variations de ce rapport.

Ces résultats n'ont malheureusement pas la précision nécessaire pour des plans rationnels de sélection. Les auteurs n'ont souvent fait que des observations phénotypiques à partir d'effectifs en général faibles. Par ailleurs les expérimentateurs n'ont pas tous suivi le même protocole de recueil des données ni observé la même périodicité des contrôles au cours de la lactation. Ces différences pourraient affecter sérieusement les valeurs génétiques estimées puisque, selon O'CoNNor et LipToN (I960), le coefficient d'héritabilité du taux de l'extrait sec dégraissé atteint 0,90 pour des contrôles journaliers contre $0,6 \mathrm{I}$ pour des contrôles tous les 28 jours. Il est

(') Les expressions : taux azoté et taux de protéines seront utilisées indifféremment, faute d'une terminologie officielle en langue française. 
raisonnable de penser qu'une variation de même grandeur se retrouverait pour les matières azotées (fraction importante de l'extrait sec dégraissé). Enfin parmi les races exploitées en France, seule la Pie-Rouge de l'Est a fait 1'objet de travaux génétiques importants sur les propriétés du lait en relation avec la fabrication de fromage (AURIOL, I954, I962).

\section{MATÉRIEI, ET TECHNIQUES}

\section{MÉTHODES DE CONTROLE E'T D'ANALYSE}

Pour les besoins de son programme de testage, la Coopérative d'Élevage du Centre Nord (C. E. C. N.) mesure la teneur en matière azotée de toutes les vaches contrôlées dans son rayon d'action (Loiret et Yonne principalement). Les contrôles sont effectués suivant les règles adoptées par le Comité National Fédératif de Contrôle Laitier. Les prélèvements mensuels de lait sont en. voyés au Laboratoire d'Amilly (Loiret) qui détermine le taux butyreux par la méthode Gerber et le taux azoté par la méthode colorimétrique au noir amido. Cette dernière opération est supervisée par la Station expérimentale laitière de Poligny (Jura). Les quantités de lait total, de matières azotées totales et de matières grasses totales ont été calculées par la méthode dite " $D$ " (CARRÉ et al., 1958).

\section{MAT'́RIEL D'ÉTUDE}

Pour éviter toute correction, seules les premières lactations d'une durée supérieure à 150 jours ont été retenues. De plus, on a éliminé les résultats de tous les groupes (filles d'un même père) n'atteignant pas l'effectif minimum de 23 sujets. Les taureaux représentés dans l'échantillon conservé appartenaient tous au C.I.A. de Charmoy (Yonne). Une description de la zone d'action et du mode de travail de ce Centre a été faite par Lesseur et al. (I959) et AUGros (I958). La répartition des lactations, l'effectif de taureaux et le nombre moyen de filles par père sont reportés globalement et pour chaque race, dans le tableau $I$.

Parce qu'elle était représentée par des effectifs trop faibles, la Brune des Alpes a été exclue de toutes les interprétations séparées par races, mais elle figure cependant dans les analyses globales.

\section{TABLEAU I}

Réparition au total et par race des effectifs de taureaux et de lactations. Nombre moyen de filles par père

\begin{tabular}{|c|c|c|c|}
\hline \multirow{2}{*}{ Race des taureaux } & \multicolumn{2}{|c|}{ Nombre de } & \multirow{2}{*}{$\begin{array}{l}\text { Nombre moyen } \\
\text { de filles par père }\end{array}$} \\
\hline & Taureaux & Lactations & \\
\hline 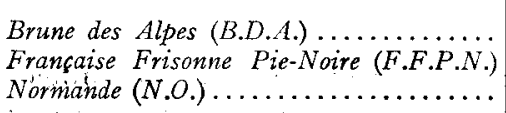 & $\begin{array}{r}3 \\
21 \\
21\end{array}$ & $\begin{array}{r}73 \\
788 \\
723\end{array}$ & $\begin{array}{l}24,3 \\
37,5 \\
34,4\end{array}$ \\
\hline Total $\ldots \ldots \ldots \ldots \ldots \ldots \ldots \ldots \ldots$ & 45 & 1584 & 1135,2 \\
\hline
\end{tabular}




\section{MÉTHOdES D'ANALYSE ET DE CALCUL}

Voici le modèle mathématique et les symboles utilisés pour l'analyse et l'interprétation des données :

$$
\text { Modèle }: y_{i j k}=\mu+r_{i}+t_{i j}+e_{i j k} \text {. }
$$

\section{SYMBOLES}

Indices : $i=\mathrm{n}^{\mathrm{o}}$ de la race du taureau;

$j=\mathrm{n}^{\mathrm{o}}$ du père à l'intérieur de la race ;

$k=\mathrm{n}^{0}$ d'une fille à l'intérieur du groupe de ses demi-sceurs.

$y_{i j k}$ : Valeur phénotypique observée pour la variable $\mathrm{Y}$.

$\mu$ : Moyenne théorique de la population pour $\mathrm{Y}$.

$r_{i}$ : Déviation moyenne théorique, autour de $\mu$, des animaux de la race $i$.

$t_{i j}$ : Déviation moyenne théorique, autour de $\mu+r_{i}$, des filles du père $i j$.

$e_{i j k}: e i j k=y_{i j k}-\left(\mu+r_{i}+t_{i j}\right)$. Variation résiduelle.

Aux 3 derniers niveaux de cette classification hiérarchique correspondent 3 composantes orthogonales de la variance dues à des différences entre races, entre descendants de taureaux intra-race et enfin entre vaches à l'intérieur d'un même groupe de demi-sœurs de père. La composante "Race" sera désignée par le symbole $\sigma_{\mathrm{R} a c c}^{2}$, la composante "Taureau " par $\sigma_{t}^{2}$ et la composante "Résiduelle » par $\sigma_{\mathrm{R}}^{2}$. Le cofficient d'héritabilité de $\mathrm{Y}\left(h^{2}\right)$ et la variance phénotypique $\left(\sigma_{\mathrm{p}}\right)$ seront toujours calculés intra-race par les formules :

$$
h^{2}=4 \frac{\sigma_{t}^{2}}{\sigma_{\imath}^{2}+\sigma_{\mathrm{R}}^{2}} \text { et } \sigma_{p}^{2}=\sigma_{t}^{2}+\sigma_{\mathrm{R}}^{2} .
$$

D'une façon tout à fait analogue, la somme des produits centrés de 2 variables $\mathrm{X}$ et $\mathrm{Y}$ se subdivise en 3 composantes orthogonales de la covariance "Race ", "Taureau ", "Résiduelle ". La covariance phénotypique, dans la race, est égale à la somme des composantes "Taureau " et " Résiduelle \%.

Les coefficients de corrélation génétique $(\rho)$ et phénotypique $(\pi)$ et le coefficient de régression génétique $\left(\beta_{\mathrm{Y} / \mathrm{X}}\right)$ de $\mathrm{Y}$ sur $\mathrm{X}$, intra-race, seront :

$$
\begin{gathered}
\rho=\frac{\text { Covariance "Taureau " }}{\sqrt{\text { Produit des variances " Taureau " }}} \text { pour } \mathrm{X} \text { et } \mathrm{Y} \\
\pi=\frac{\text { Covariance phénotypique }}{\sqrt{\text { Produit des variances phénotypipues }}} \text { pour } \mathrm{X} \text { et } \mathrm{Y} \\
\beta_{\mathrm{Y} / \mathrm{X}}=\frac{\text { Covariance " Taureau " pour } \mathrm{X} \text { et } \mathrm{Y}}{\text { Variance "Taureau " pour } \mathrm{X}}
\end{gathered}
$$

Leurs valeurs estimées, à partir des données analysées seront désignées par les lettres latines correspondantes. Les composantes de la variance et de la covariance ont été calculées par la méthode de GaNGULI applicable au cas de schémas hiérarchiques non-orthogonaux (VISSAC, 1962). Pour que ces estimées et celles qui en dérivent soient statistiquement correctes, il faudrait que tous les éléments du modèle mathématique, sauf $\mu$, se répartissent suivant 3 lois de distribution indépendantes des indices $i, j, k$; et d'espérance mathématique nulle. L'exactitude de ces hypothèses sera discutẻe dans l'exposé des résultats.

\section{RÉSULTATS ETT DISCUSSION}

\section{I. - PARAMÈTRES STATISTIQUES ÉLÉMENTAIRES}

Pour chaque variable centrée et normée la courbe des fréquences cumulées relatives a été tracée. Le test approximatif de SMrRnow (Drxon et MAssey, I957) appliqué à chaque variable n'a pas mis en évidence de différence significative, au seuil de 5 p. Ioo, entre les fonctions de distribution des races F.F.P.N. ou Nor- 
mande. Les courbes des différentes variables, sauf pour la durée de lactation, étant elles-mêmes très proches, on n'a reproduit que 2 courbes moyennes, l'une pour la durée et l'autre pour tous les autres critères (fig. I). L'abscisse de ce graphique est graduée en $\mathrm{I} / 4$ d'écart-type autour de la moyenne de chaque variable. Par rapport à la loi normale, on relève un déficit dans les valeurs extrêmes et une légère dissymétrie à gauche de la courbe.

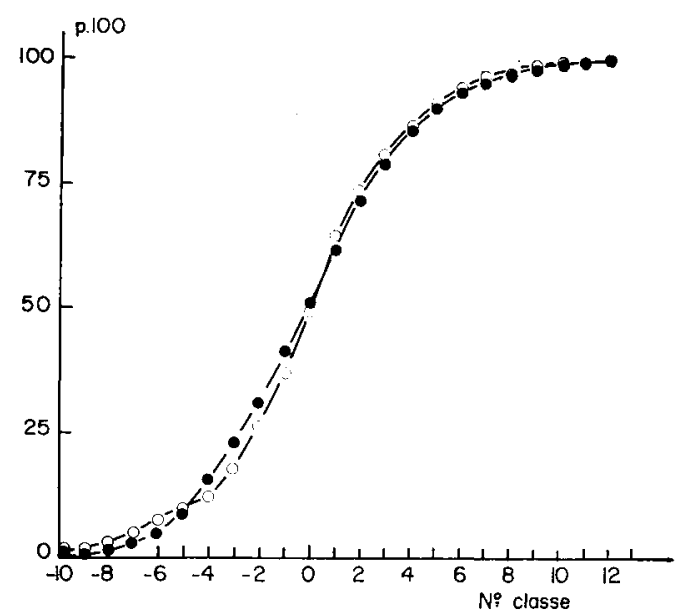

FIG. I. - Fréquences relatives cumulées des variables ramenées à leur moyenne propre et exprimées en unités de $1 / 4$ d'écart-type. Moyenne des courbes pour les races F.F.P.N. et Normande.

- - D Durée de lactation.

- Q Quantité de lait, taux de protéines et taux butyreux.

Une comparaison de l'amplitude de variation entre les taux azoté et butyreux est fournie par les limites de 1'intervalle qui contient le tiers des données de chaque côté de la moyenne et par l'étendue de cet intervalle (tab1. 2).

Les limites et les étendues sont plus élevées en race Normande, tandis que le taux de protéines apparait toujours moins fluctuant autour de sa moyenne que le taux butyreux

\section{TABLEAU 2}

Limites entre lesquelles se situent, autour de la moyenne, les $2 / 3$ des données.

L'étendue égale la différence entre ces limites.

La ligne $T A / T B$ est obtenue par division des éléments correspondants des lignes taux azoté et taux butyreux

\begin{tabular}{|c|c|c|c|c|}
\hline \multirow{2}{*}{ Race } & \multicolumn{2}{|c|}{$H . F . P . N$} & \multicolumn{2}{|c|}{ Normande } \\
\hline & Linites & Étendue & Iimites & Etendue \\
\hline 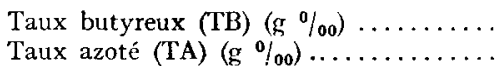 & $\begin{array}{l}3: 3,0-38,6 \\
28,9-32,8\end{array}$ & $\begin{array}{l}5,6 \\
3,9\end{array}$ & $\begin{array}{l}35,3-41,9 \\
31,1-35,3\end{array}$ & $\begin{array}{l}6,6 \\
4,2\end{array}$ \\
\hline $\mathrm{TA} / \mathrm{TB}(\times 100)$ & $\ldots$ & 69 & - & $\sigma_{t}^{\prime}$ \\
\hline
\end{tabular}


TABLEAU 3

Moyennes, écarts-types phénotypiques et coefficients de variation phénotypiques par race. La ligne $T A / T B$ vient de la division (dans chaque colonne) des lignes taux azoté et taux butyreux, de même la ligne $M A / M G$ vient de la division des lignes matière azotée totale et matière grasse totale.

Les écarts-types et coefficients de variation sont arrondis $\dot{a} 2$ ou 3 chiffres significatifs

\begin{tabular}{|c|c|c|c|c|c|c|}
\hline Race & \multicolumn{3}{|c|}{$F . F . P . N$} & \multicolumn{3}{|c|}{ Normande } \\
\hline Variable & Moyenne & Écart-type & $\begin{array}{c}\text { Coefficient } \\
\text { de variation } \\
(\%)\end{array}$ & Moyenne & Écart-type & $\begin{array}{c}\text { Coefficient } \\
\text { de variation } \\
(\%)\end{array}$ \\
\hline Durée (jours) ............. & 298 & 56 & 19 & 289 & 55 & 19 \\
\hline Lait total $(\mathrm{kg}) \ldots \ldots \ldots \ldots \ldots$ & 3480 & 960 & 28 & 2690 & 810 & 30 \\
\hline Matière grasse totale $(\mathrm{MG})(\mathrm{kg})$ & 124 & 36 & 29 & 104 & 33 & 31 \\
\hline Matière azotée totale (MA) (kg) & 108 & 31 & 29 & 90 & 27 & 30 \\
\hline $\begin{array}{c}\text { Taux butyreux moyen }(\mathrm{TB}) \\
(\mathrm{g} \% \mathrm{oo}) \ldots \ldots \ldots \ldots \ldots \ldots \ldots\end{array}$ & 35,9 & 2,97 & 8,28 & 38,6 & 3,45 & 8,92 \\
\hline Taux azoté moyen (TA) (g \%oo) & 31,0 & 1,98 & 6,38 & 33,4 & 2,14 & 6,40 \\
\hline $\mathrm{TA} / \mathrm{TB}(\times 100) \ldots \ldots \ldots \ldots$ & 86,5 & 67 & 77 & 86,5 & 62 & 72 \\
\hline $\mathrm{MA} / \mathrm{MG}(\times 100) \ldots \ldots \ldots \ldots$ & 86,5 & 85 & 98 & 86,5 & 84 & 97 \\
\hline
\end{tabular}

Les valeurs obtenues pour les paramètres typiques des distributions (moyenne, écart-type phénotypique, et coefficient de variation) sont rassemblées dans le tableau 3 .

Comparer les races d'après ces résultats n'offrirait d'intérêt que si elles étaient exploitées, en moyenne, dans le même miliet1, ce qui ne correspond vraisemblablement pas à la réalité. On notera cependant la supériorité des Normandes à la fois pour les taux azoté et butyreux, le rapport de ces taux étant par ailleurs identique, à celui des Frisonnes. Pour celles-ci les résultats sont à rapprocher de ceux enregistrés en Hollande pour les vaches contrôlées durant l'année I96I (5) : $4420 \mathrm{~kg}$ de lait à 38,5 p. I ooo de taux butyreux et 33,4 p. I ooo de taux azoté en 306 jours. En I959, les vaches Normandes contrôlées en France ont produtit $3650 \mathrm{~kg}$ de lait à 4I,2 p. I 000 de taux butyreux en 288 jours (C. F. N. C. L. (7)). Les raisons pour lesquelles les teneurs sont manifestement plus faibles dans notre échantillon ne sont pas entièrement connues. L'âge des animaux ( ${ }^{\text {res }}$ lactations pour la C. E. C. N. ; toutes lactations ailleurs) ne joue sans doute qu'un rôle minime. Il n'explique en effet, que 5 p. Ioo de la variance au plus et tendrait à majorer les résultats du Centre de Charmoy car les taux diminuent avec l'âge (RoBERTson et al., I956, Von KRosigket al., I960). Des écarts aussi grands, entre notre échantillon et ceux des berceaux de race, pour le taux azoté $(-2,4$ p. I ooo F.F.P.N.) et le taux butyreux ( $-2,6$ p. I ooo dans les 2 races) ne s'expliquent sans doute pas en entier par des différences génétiques. Dans la région de la $\mathrm{C}$. F. C. N. on achète en effet depuis longtemps la plupart des taureaux dans les berceaux d'élevage et le peuplement frison s'est fait récemment à partir de souches importées des Pays-Bas. Il paraît donc raisonnable d'admettre 
que le milieu local défavorise la production d'un lait à concentration élevée. En Montbéliarde, seule race française bien connue (Auriol, I962 b), les taux azoté (3I,8 p. I ooo) et butyreux $(36,5$ p. I ooo) moyens sont légèrement supérieurs à ceux des F.F.P.N. de Charmoy.

Écarts-types et moyennes tendent à varier dans le même sens. Ces paramètres sont plus élevés en race F.F.P.N., pour les quantités, et en race Normande pour les taux. En race Normande, les coefficients de variation restent toujours un peu plus grands traduisant ainsi une certaine hétérogénéité phénotypique. D'après le même critère les variables se classent aisément en 3 groupes de variabilité décroissante : d'abord les quantités, ensuite la durée de lactation, enfin les taux. La très grande mobilité des quantités s'explique d'ailleurs en partie par celle de la durée car on verra plus loin que ces caractères sont liés. Le taux azoté varie lui-même nettement moins que le taux butyreux. Le rapport (TA/TB) des écarts-types, plus faible en race Normande, est en moyenne inférieur à $2 / 3$ et le rapport des coefficients de variation à $3 / 4$. Ces valeurs concordent bien avec celles obtenues par AURIoL et JARRIGE (I962 a) à partir, il est vrai, de données d'origines assez disparates. La plus grande stabilité des constituants azotés du lait se retrouve quand on compare les quantités de matières produites mais de façon moins nette : le rapport de leurs écarts-types est roisin de $5 / 6$ et leurs coefficients de variation presque identiques.

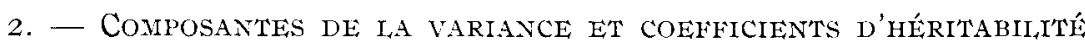

La variance phénotỹpique a été décomposée en 3 parties attribuées à des différences entre races, entre les taureaux d'une même race et enfin à des variations résiduelles entre les filles d'un même taureau. La même analyse a été répétée par race, la composante race ne pouvant, bien entendu, être alors estimée. Les racines carrées de ces valeurs arrondies à 2 chiffres significatifs, se trouvent dans le tableau 4 .

Dans le tableau 5 se trouvent rassemblées les valeurs des coefficients d'héritabilité, de leur écart-tỹpe estimé et enfin les coefficients d'héritabilité après élimination totale de l'influence de la durée par analyse de covariance. Les écarts-types ont été estimés à partir de la formule donnée par FALCONER (I960), en prenant comme effectif par taureau, supposé constant, la moyenne harmonique du nombre de filles par père.

La composante "Race " de la variance n'estime pas correctement la part de la variation qui a sa source dans cles différences génétiques entre races. Elle mesure, en réalité, les effets conjoints pour les populations étudiées tant de leur dissemblance génétique que des différences dans les conditions de leur exploitation. De plus F.F.P.N. et Normande appartiennent à 2 types d'animaux bien distincts et ne peuvent être considérées comme choisies " au hasard " dans l'ensemble des races. Malgré ces réserves il convient de remarquer que l'efficacité de la sélection diminue, en apparence, quand on néglige cette source importante de variation dans les analyses faites intra-race. Par exemple le coefficient d'héritabilité de la quantité de lait double si on inclut la composante race dans la variance génétique et phénotypique.

Les quantités de lait, de matière azotée totale et de matière grasse totale se comportent pratiquement de la même façon avec seulement un coefficient d'héri- 


\section{TABLEAC 4}

Racines carrées des composantes de la variance protenant de différences entre races, entre taureaux et de causes résiduelles.

Ces valeurs sont données avec un arrondi à 2 chiffres significatifs.

La ligne TAITB a été obtenue par division, dans chaque colonne, des lignes taux asoté et taux butyreux. et la ligne $M A / M C$ par division des lignes matière asotée totale et matiere grasse totale

\begin{tabular}{|c|c|c|c|c|c|c|c|}
\hline Race & & Toutes rac & & $F . I$ & $P . X$ & Sor & nande \\
\hline Source de variation & Race & Taureau & Résiduelle & Taureau & Residuelle & Taureau & Résiduelle \\
\hline Durće (jours) . . . . . . . & 4,9 & 8,5 & $\pi$ & 5,10 & 5.5 & $9,{ }^{\prime}$ & 51 \\
\hline Lait $(\mathrm{kg}) \ldots \ldots \ldots \ldots \ldots$ & 520 & $\underline{900}$ & 870 & $\because 20$ & ?'1! & 180 & 790 \\
\hline $\begin{array}{l}\text { Matiere grasse totale (MG } \\
(\mathrm{kg}) \ldots \ldots \ldots \ldots \ldots \ldots \ldots\end{array}$ & $1^{\prime} \mathbf{t}$ & $7, \pm$ & $33^{\prime}$ & 7,0 & 85 & 6,7 & $3:$ \\
\hline $\begin{array}{r}(\mathrm{kg}) \ldots \ldots \ldots \ldots \ldots \ldots \\
\text { Taux butyreux moyen (TB) }\end{array}$ & 12 & $i, 1$ & $\because 9$ & 6,6 & $: 80$ & 5,6 & 27 \\
\hline$(g \%$ \% $\ldots \ldots \ldots \ldots \ldots$ & 1,8 & 0,79 & 3,1 & $\left(1, e^{\prime} t\right.$ & 2,9 & 1.0 & $: 3,3$ \\
\hline $\begin{array}{l}\text { Taux azoté moyen (TA) } \\
(\mathrm{g} \% \text { oo }) \ldots \ldots \ldots \ldots \ldots \ldots\end{array}$ & 1,6 & 0,57 & $\because, 0$ & 0,11 & $1,: 1$ & 0,69 & 2,0 \\
\hline $\mathrm{TA} / \mathrm{TB}(\%) \ldots \ldots \ldots \ldots$ & 89 & $7:$ & iis & 89 & lit; & 69 & 61 \\
\hline $\mathrm{MA} / \mathrm{MG}(\%) \ldots \ldots \ldots \ldots \ldots$ & 86 & 85 & $x^{\prime}$ & 57 & $\times 1 ;$ & 8:3 & $81 t$ \\
\hline
\end{tabular}

\section{TABLEAC 5}

Coefficients d'héritabilité $\left(h^{2}\right)$ et leurs écarts-types estimés $\left(\sigma_{h^{2}},\right)$

\begin{tabular}{|c|c|c|c|c|c|c|c|}
\hline \multirow[t]{2}{*}{ Race } & \multicolumn{2}{|c|}{ 'loutes races } & \multicolumn{2}{|c|}{ Nornumle } & \multicolumn{2}{|c|}{$F . F . P . \triangle$} & \multirow{2}{*}{$\begin{array}{c}\text { Joutes races } \\
\text { a durée } \\
\text { cle lactation } \\
\text { constante }\end{array}$} \\
\hline & $h^{2}$ & $\sigma_{h^{2}}$ & $h^{2}$ & $\sigma_{h^{2}}^{2}$ & $h^{2}$ & $\sigma_{h^{2}}$ & \\
\hline Durée..$\ldots \ldots \ldots \ldots \ldots$ & 0,09 & 0,05 & $0,1:$ & 0.07 & $0,0_{1}^{\prime}$ & 0,05 & \\
\hline Lait total $\ldots . . . \ldots \ldots$ & 0,20 & 0,06 & $0, \because 0$ & $0,11)$ & 0,20 & 0,10 & 0,15 \\
\hline Matière grasse totale ..... & 0,18 & 0,06 & 0,17 & 0,09 & 0,18 & 0,09 & 0.12 \\
\hline Matière azotée totale . . . . . & 0,17 & $0,0 t$ & $0,11 i$ & $0.0 ! 1$ & 0.19 & 0,09 & 0,12 \\
\hline Taux butyreux moyen ... . . & $0, \because 21$ & 0,07 & $(1,1 ; \pi$ & $0,1^{\prime} \mathbf{t}$ & 0.10 & 0,07 & $0, \because 5$ \\
\hline Taux azoté moyen & 0,31 & 0,08 & $0,4: 2$ & 11,15 & 0,17 & 0,09 & 0,32 \\
\hline
\end{tabular}


tabilité un peu plus fort pour la production de lait (tabl. 5). Les écarts-types génétiques de la Frisonne dépassent ceux de la Normande (tab1. 4) de sorte que le coefficient d'héritabilité reste finalement presque constant entre races. Il en est de même pour les rapports des écarts-types génétiques des productions de matière azotée et de matière grasse qui restent, d'ailleurs, très proches de leurs valeurs " résiduelle" ou "phénotypique ". Les coefficients d'héritabilité de la production de lait sont un peu plus faibles que la valeur généralement admise bien que les écarts-types génétiques soient du même ordre de grandeur que ceux trouvés par RoBERTson et al. (I956) et O'ConNor (I958).

Pour les taux, l'analyse des résultats révèle un contraste frappant entre les 2 races. Les coefficients d'héritabilité des Normandes pour le taux azoté $(0,42)$ et 1e taux butyreux $(0,37)$ sont à rapprocher des valeurs calculées par la même méthode. Auriol (I962 b) trouve 0,42 et 0,39 sur 329 Montbéliardes, PolrTrEK (I957) 0,45 et 0,79 sur I8o Frisonnes, Von Krosigk et al. (r960) 0,44 et 0,49 à partir de 793 données. La concordance reste bonne avec les auteurs qui ont éliminé l'influence de l'étable pour les comparaisons mère-fille. Pour RoBertson et al. (I956) les coefficients d'héritabilité des taux azoté et butyreux sont 0,48 et 0,32 (500 couples en race Ayrshire) et pour von Krosigk et al. (I g60) 0,48 et 0,52 . Cependant la valeur généralement admise pour le taux butyreux $(0,50)$ dépasse le chiffre obtenu ici. D'après PoLy et VISSAC (I959) la différence viendrait de la sélection sur ascendance des taureaux. En effet cette sélection réduisant la variance génétique sans modifier beaucoup la variance phénotypique diminuerait l'héritabilité, calculée à partir de leur rapport. Sur des données enregistrées par la C. E. C. N. de I95 I à I957 ces auteurs ont estimé 1'héritabilité du taux butyreux à 0 , I9 d'après la ressemblance des demi-sœurs de père mais à 0,66 d'après la régression des filles sur leur mère. Or les taureaux du Centre de Charmoy sont choisis avec soin d'après la richesse en graisse du lait de leurs ascendants alors que les éleveurs n'éliminent presque pas leurs vaches d'après ce critère. Une autre confirmation de cette thèse est fournie par les résultats obtenus ici en race F.F.P.N. : les héritabilités sont faibles parce que les variances "Taureau " le sont aussi. En effet, les variances résiduelles vraies (variance "Résiduelle »variance "Taureau "), très proches pour le taux butyreux, sont pratiquement identiques pour les 2 races dans le cas du taux de protéines. Sans négliger les causes d'erreur purement statistiques de ces estimées, il paraît ainsi raisonnable d'admettre une sélection plus intense sur le taux butyreux en race Frisonne. Une telle sélection pourrait d'ailleurs être volontaire ou non, l'échantillonnage d'un nombre petit d'animaux étant évidemment sujet à d'importantes fluctuations aléatoires.

Le rapport (TA/TB) des écarts-types génétiques du taux azoté au taux butyreux est, de loin, le plus élevé en race Frisonne (o,89). Il dépasse alors largement le rapport des écarts-types phénotypiques $(0,67)$ (tab1. 4 et 3 ). Au contraire toutes ces valeurs concordent de façon satisfaisante pour les quantités de matière azotée ou de matière grasse. Dans une étude ultérieure on montrera que ceci indique une intensité de sélection plus élevée sur le taux butyreux en F.F.P.N. Dans le cas de la quantité de matière grasse la sélection sur descendance n'aurait eu qu'une efficacité limitée. 


\section{3. 一 Corrélations phénotypiques eit GÉNÉtrques}

Ia composante "Race " explique une part importante de la covariance totale. Cependant cette source de covariation a été éliminée et les calculs faits "intra-race " pour les mêmes raisons que dans le cas des composantes de la variance. A remarquer au passage que 2 races seulement avaient des effectifs suffisants pour modifier en pratique les résultats des calculs. En pareil cas, le coefficient de corrélation obtenu à partir des composantes "race " de la covariance et de la variance doit varier autour de $\pm I$, ce qui s'est effectivement produit pour cet échantillon (tab1. 6).

\section{TABIEAU 6}

Coeffrients de corrélation phénotypiques (au-dessus de la diagonale) et génétiques (sous la diagonale) arrondis à la $2^{\mathrm{e}}$ décimale. Dans chaque case les résultats se lisent de haut en bas pour : les F. F.P. N., Normandes et toutes races, dans l'ordre

\begin{tabular}{|c|c|c|c|c|c|c|}
\hline & \multicolumn{6}{|c|}{ Les coefficients phénotypiques sont au-dessus de la diagonale } \\
\hline Variables & Duréa & Lait total & $\begin{array}{c}\text { Matière } \\
\text { grasse totale }\end{array}$ & $\begin{array}{c}\text { Matière } \\
\text { azotée totale }\end{array}$ & $\begin{array}{l}\text { Taux buty- } \\
\text { reux moyen }\end{array}$ & $\begin{array}{c}\text { Taux azoté } \\
\text { moyen }\end{array}$ \\
\hline Durée & & ${ }_{0,67}^{0,66} 0$ & $\begin{array}{ll}0,67 & \\
0,68 & 0,69\end{array}$ & $\begin{array}{ll}0,67 & \\
& 0,68 \\
0,68 & \end{array}$ & $\begin{array}{ll}0,14 & \\
0,14 & \end{array}$ & $\begin{array}{ll}0,14 & 0,06 \\
0,09 & \end{array}$ \\
\hline Lait total & $\begin{array}{l}>1 \\
\quad 0,87^{0,84}\end{array}$ & - & $\begin{array}{ll}0,96 & \\
0,96 & 0,96\end{array}$ & $\begin{array}{ll}0,98 & \\
0,98 & 0,98\end{array}$ & $\begin{array}{l}-0,03 \\
-0,01\end{array}$ & $\begin{array}{l}-0,01 \\
-0,02\end{array}$ \\
\hline Matière grasse totale & ${ }_{0,88}^{0,94}$ & $\begin{array}{ll}0,97 & \\
0,95 & 0,90\end{array}$ & - & ${ }_{0,97}^{0,97}$ & ${ }_{0,27}^{0,25}$ & $\begin{array}{ll}0,16 & \\
0,14 & 0,12\end{array}$ \\
\hline Matière azotée totale & $\begin{array}{ll}0,97 & \\
0,88 & 0,92\end{array}$ & $\begin{array}{ll}0,98 & \\
0,96 & 0,95\end{array}$ & $\begin{array}{l}0,97 \\
0,96\end{array}$ & & $\begin{array}{ll}0,10 & \\
0,11 & \end{array}$ & ${ }_{0,19}^{0,18}$ \\
\hline $\begin{array}{c}\text { Taux butyreux } \\
\text { moyen }\end{array}$ & $\begin{array}{cc}-0,89 & \\
0,01 & \end{array}$ & $\begin{array}{l}-0,14 \\
-0,22\end{array}$ & $\begin{array}{ll}0,10 & \\
0,10 & \end{array}$ & $\begin{array}{l}-0,03 \\
-0,06\end{array}$ & & $\begin{array}{ll}0,61 & \\
0,54 & 0,50\end{array}$ \\
\hline \multirow[t]{2}{*}{$\begin{array}{l}\text { Taux azoté } \\
\text { moyen }\end{array}$} & $\begin{array}{l}-0,77 \\
-0,13\end{array}$ & $\begin{array}{l}-0,10 \\
-0,39 \\
-0,25\end{array}$ & $\begin{array}{l}-0,01 \\
-0,07\end{array}$ & $\frac{0,12}{-0,02}-0,08$ & $\begin{array}{ll}0,46 & \\
0,57 & 0,63\end{array}$ & \\
\hline & \multicolumn{6}{|c|}{ Les coefficients génétiques sont au-dessous de la diagonale } \\
\hline
\end{tabular}

\section{I) Corrélations phénotypiques}

Les corrélations phénotypiques (tab1. 6) sont très homogènes d'une race à l'autre. Pour le taux de protéines, cependant, les valeurs apparaissent légèrement plus élevées, pour les F.F.P.N.

Les 3 quantités se comportent de la même façon. Elles dépendent nettement de la durée $(r=0,68)$ et surtout elles se déterminent mutuellement en très grande 
partie $(r=0,98)$. La liaison lait total et matière grasse ou matière azotée totales ne varie pratiquement pas à durée de lactation constante ; elle peut donc être étudiée en elle-même sans référence à l'action de la durée. Du point de rue phénotypique il importe finalement assez peu de connaitre le taux azoté ou butyreux pour prévoir les quantités de matières azotées ou grasses puisque les coefficients de corrélation directe ne sont que de +0 , I9 et $+0,27$ et les valeurs croisées de +0, I I et +0 , I 4 .

Les teneurs ont tendance à augmenter pour les lactations de longue durée, ce qui est logique, et sont quasi indépendantes de la quantité de lait. A durée de lactation constante cependant, une corrélation phénotypique négative apparaît pour les 2 taux $(r=-0, I 6$ par exemple en race F.F.P.V.). Ces résultats ne nécessitent pas d'autres commentaires car ils confirment les nombreuses études qui ont été consacrées à ce sujet. La valeur moyenne, intra-race, du coefficient de corrélation entre le taux azoté et le taux butyreux $(r==+0,54)$ coïncide elle-même avec celle obtenue par AURIOL et JARRIGE (I962a) à partir de données bibliographiques. Sans nier l'existence possible de différences raciales, il paraît donc raisonnable d'admettre que le coefficient de corrélation phénotypique pour les taux est compris entre 0,50 et 0,60 . Le coefficient de corrélation partielle entre matière grasse totale et matière azotée totale, à quantité de lait constante, a été estimé à $+0,53$ d'après les valeurs moyennes obtenues dans notre échantillon. La corrélation entre les taux et la quantité de lait étant pratiquement nulle, il s'agit d'une autre estimation de la corrélation des taux entre eux qui confirme la valeur trouvée directement. Il en est d'ailleurs de même pour les corrélations partielles, à quantité constante de lait, entre la matière azotée et le taux butyreux $(r=+0,60)$ et vice versa $(r=0,57)$. La moyenne de ces valeurs $(r=+0,56)$ peut ainsi être prise comme une estimée de la corrélation phénotypique entre les taux.

\section{2) Corrélations génétiques}

La variance génétique de la durée de lactation ne diffère pas significativement de 0 , surtout pour la race F.F.P.N. Les corrélations génétiques de cette variable ne seront donc pas discutées ici. Pour les autres critères, les 2 races fournissent des estimées dont les valeurs s'accordent bien, surtout si l'on tient compte de leurs intervalles de confiance (tab1. 6). Une réserve est cependant à faire : en race $F . F . P . N$. toutes les corrélations sont supérieures en valeur algébrique. Un classement s'impose ici aussi, qui regroupe les quantités produites d'une part et les taux de l'autre.

Le coefficient de corrélation génétique moyen $(r=0,57)$ entre les taux est légèrement supérieur au coefficient phénotypique $(r=0,54)$ comme l'avaient noté von KROSIGK et $a l$. (I 960 ). Ces auteurs avaient estimé ce paramètre à 0,70 d'après les résultats moyens fournis par l'analyse des relations entre 240 couples mère-fille $(r=0,62)$ et entre 232 groupes de demi-sœurs $(r=0,78)$. Le premier de ces résultats est très près de celui calculé pour la race Normande $(r=0,63)$, tandis que la valeur en race Frisonne $(r=0,46)$ est comparable à celle obtenue par RoBERTson et al. (I958) pour des vaches Ayrshire $(r=0,48)$.

On sait depuis longtemps qu'il existe une corrélation génétique négative entre quantité de lait et taux butyreux. D'après Gaines (I940) cette relation serait d'autant plus étroite que la teneur moyenne pour la race est plus élerée. C'est effectivement ce que confirme la comparaison des résultats de l'échantillon étudié ici : pour 
les Frisonnes $r=-$ o, I4 et pour les Normandes $r=-0,35$. RoBERTSON et al. (1956) ont calculé un coefficient de corrélation génétique positif entre quantité de lait et teneur en matières azotées totales. Avec des animaux de la même race O'ConNor (I958) a cependant trouvé une liaison génétique négative entre quantité de lait et teneur en extrait sec dégraissé (constitué, rappelons-1e, pour une part importante par les matières azotées). Pour l'échantillon analysé les valeurs sont pratiquement les mêmes que pour les relations lait et taux butyreux, qu'on les prenne race par race ou globalement. Compte tenu ici encore de l'intervalle de confiance des estimées, il semble donc raisonnable d'admettre que les corrélations quantité de lait - taux azoté et quantité de lait - taux butyreux sont du même ordre de grandeur.

Les coefficients de corrélation génétiques entre les quantités restent presque aussi élevés que les valeurs phénotypiques. En particulier, la variance génétique de la quantité de matière azotée est réduite de $90 \mathrm{p}$. Ioo au moins quand on connaît la production de lait ou de matière grasse. Par contre, les corrélations génétiques entre les quantités de graisse ou de matières azotées et les taux homologues sont très faibles. Il existe même une relation négative entre quantité de matière azotée et taux butyreux et vice versa. Aussi une sélection sur le taux butyreux aboutirait-elle à diminuer un peu le potentiel de production des matières azotées. Cette situation s'explique par le jeu des corrélations négatives entre taux et quantité de lait. En effet, les corrélations partielles, à quantité de lait constante, ont été estimées d'après les valeurs obtenues, en moyenne, dans notre échantillon :

entre matière azotée et taux butyreux $r=+0,55$

entre matière grasse et taux azoté $\quad r=+0,77$

Leur moyenne $(r=+0,66)$ égale presque le coefficient de corrélation partielle $(r=+0,67)$ entre matière grasse et matière azotée, toujours en éliminant l'effet de la quantité de lait. Malgré leur imprécision, ces corrélations partielles sont intéressantes, parce qu'elles fournissent une autre estimation des liaisons entre constituants, après élimination de l'effet quantité de lait. La corrélation moyenne entre les taux devrait donc leur être supérieure car ils sont liés par 1'intermédiaire de la quantité de lait. Cela se produit effectivement en race Frisonne $(r=0,43$ contre 0,46$)$ mais non en Normande (o,69 contre 0,63$)$, les différences, il est vrai, n'ayant sans doute pas de signification statistique. Ces résultats tendent à montrer qu'il existe entre les taux une corrélation génétique supérieure, en moyenne, à o,6o.

\section{CONCLUSION}

Cette étude a permis de compléter, pour deux des plus importantes races françaises, les enquêtes déjà faites dans le Jura sur la race Montbéliarde. Ėlle nous a fourni les estimations des paramètres indispensables pour apprécier l'efficacité d'une sélection sur les constituants azotés du lait. (Coefficients d'héritabilité, composantes de la variance, corrélations génétiques et phénotypiques). Cependant les valetiis ont pu être faussées par le type de sélection pratiqué par le Centre de Charmoy, comme nous l'avons relevé au cours de la discussion. 
Par ailleurs l'opportunité du dosage des matières azotées, dans les échantillons individuels prélevés lors du contrôle laitier, doit être jugée dans le cadre d'une sélection portant sur tous les critères de production à la fois. Pour le testage, le choix des reproducteurs se fait, en effet, par un jugement synthétique qui sera en définitive bon s'il permet d'améliorer la rentabilité de la production laitière. Nous nous proposons de discuter plus en détail certains de ces points dans une étude ultérieure.

Reçu pour publication en mars 1964 .

\section{SUMMARY}

\section{PROGENY TESTING OF DAIRY BULLS FOR PROTEIN PRODUCTION. PRELIMINARY RESULTS}

An analysis of 1584 first lactation records, from 45 bulls of the Charmoy $A$. I. center, was undertaken. The animals belonged mainly to the French Friesian (F. F. P. N. : 788 १९, 21 ( $0^{\circ}$ ) and the Normandy (NO. : 724 우, 2 I ơ $0^{*}$ ) breeds.

The mean and the limits including $2 / 3$ of the data around the mean, for the protein (TA) and butteríat (TB) percentage were :
F.F.P.N.
TA p. I 000 3I.0; $28.9-32.8$
TB p. I $00035.9 ; 33.0-38.6$
No.
$33.4 ; 31 . \mathrm{I}-35.3$
$38.6 ; 3.5 \cdot 3-41.9$

There is a great variability (C. V. $\geqslant 3 \circ \mathrm{p}$. I00) in the values of total milk yield, butterfat contents (MG) and protein contents (MA) per lactation, the three variables being furthermore closely correlated to one another (phenotypic $r \geqslant 0.95$; genetic $r \geqslant 0,90$ ). Heritabitity values are slightly higher for milk yield but of the same order for all three variables $\left(0.16 \leqslant h^{2} \leqslant 0.20\right)$. The existing phenotypic variability of milk yield is due, in part, to lactation length $(r \geqslant 0.67)$.

The variability of the composition elements is distinctly lower (c. $\mathrm{V} . \mathrm{:}: \mathrm{TA}=6.4{ }^{\mathrm{T}} \mathrm{TB}=8.6$ ) and the heritability values comparable but showing important breed differences (F.F. P. N. : $h^{2}$ TA $=0.17 ; h^{2} \mathrm{~TB}=0.10$. NO. $\left.: h^{2} \mathrm{TA}=0.42 ; h^{2} \mathrm{~TB}=0.37\right)$. The phenotypic and genetic correlations evaluated between butterfat and protein percentage are $0.6 \mathrm{I}$ and 0.46 for the French Friesian and 0.50 and 0.63 for the Normandy breed, respectively; the intra-breed averages are 0.54 and 0.57 .

The phenotypic correlations between the two composition elements and lactation length are very Iow $(r \mathrm{TA}=0.09 ; r \mathrm{~TB}=0 . \mathrm{I} 4)$ and practically naught between the two percentages and milk yield, except when corrected to constant lactation length $(r-0.16)$. There is little report between protein contents and percentage $(r=0.19)$ and even less between protein contents and butterfat percentage $(r=-0.06)$. A similar tendency was observed between milk yield and butterfat - protein percentage $(r \mathrm{~TB}=0.27 ; r \mathrm{TA}=0.14)$. The genetic relationship between protein contents and percentage as well as between butterfat contents and percentage is small $(r$ TA. MA $=$ $0.02 ; r \mathrm{CB} \cdot \mathrm{MG}=0.10)$; the crossed genetic correlations are negative $(r \mathrm{TA} . \mathrm{MG}=-0.07$; $r$ TB. MA $=-0.06$ ). More negative correlations between milk yield and composition were obtained for the Normandy $(r \mathrm{TA}=-0.39 ; r \mathrm{~TB}=-0.35)$ than for the French Frisian breed $(r \mathrm{TA}=$ $-0.10 ; r \mathrm{~TB}=-0.14)$.

The genetic standard deviation is lower for protein than butterfat contents (MA/MG $=0.84)$, it is also lower for protein percentage as compared to butterfat percentage (TA/TB :F.F.P.N. $=$ $0.89 ; N O .=0.69$ ). By the use of partial correlations. corrected to constant milk yield, a phenotypic correlation of 0.57 was reestimated between butterfat and protein percentages the genetic correlation being over 0.67 . Selection effects on these results and the value of a protein control will be investigated in a future study.

\section{REMERCIEMEN'TS}

Nous tenons à remercier la Coopérative d'Élevage du Centre Nord pour le très important travail technique qu'elle a réalisé et plus spécialement $M$. Chevaldonne, Directeur général, qui a mis à notre disposition tous les documents nécessaires à cette étude. 


\section{RÉFÉRENCES BIBIIOGRAPHIQUES}

Augros R., 1958. Observations sur la méthode de testage des taureaux mise en œuvre par l'Union des Coopératives Agricoles d'élevage de l'Yonne et du Loiret. Bull. Techn. Int. Ing. Serv. Agric., 127, 67-73.

Auriol P., 1954. Progeny-test et amélioration des aptitudes fromagères de la race Pie Rouge de l'Est. Bull. Techn. Inf. Ing. Serv. Agric., 87, 1-20.

Auriol P., I962. Conment améliorer à la production la teneur du lait en protéines? Bull. Techn. Int. Ing. Serv. Agric., 168, 357-374.

Auriol P., Jarrige R., ig62. Possibilités de modifier la composition du lait. Bull. Techn. Féd. Int. Lait, II, I-39.

Bureau central du Service de contrôle laitier des Pays-Bas, ig6r. Rapport Annuel.

Carré J., Poly J., Vissac B., I958. Étude des méthodes de détermination des performances laitières. Ann. Zootech., 7, $2+3-280$.

Comité Fédératif national de Contrôle laitier, 1959. Moyenne des résultats transmis aux Livres Généalogiques.

DiXoN W. I. and MAssey F. J., 1957. Introduction to statistical analysis. 2nd edition, McGraw-Hill Book Company, Inc, New York.

FALCONER D. S., I960. Introduction to quantitative genetics. Oliver and Boyd, Edinburgh.

Hancock J., 1953. Studies on monozygotic cattle twin. VII. - The relative importance of inheritance and environment in the production of dairy cattle. N. Z. J. Sci. Tech. A, 35, 67-92.

Johansson Y., г96r. In Genetic. Aspect of dairy Cattle Breeding. University of Illinois Press, I65-181

LeGates J. E., I960. Genetic and Environmental Factors affecting the Solids-non-fat Composition of Milk. J. Dairy Sci., 43, $1527-153^{2}$.

Lesseur D. et al., 1959. Problèmes pratiques posés par le testage des taureaux de race laitière. Bull. Tech. Inf. Ing. Serr. Agric., 145, 789-8I1.

Lolkema H., Van der Have A. J., Ig6o. Neth. Milk. Dairy J., 14, 277 ; cité par Auriol et Jarrige.

O'Connor L. R., 1958. The Inheritance of milk quality. C. R. XV e Cong. Int. Lait, 1, 158-63.

O'CONNOR L. K. and LIPTON S., I960. The effect of various sampling intervals on the estimation of lactation milk yield and composition. J. Dairy Res., 27, 389-398.

Politiek R. D., I957. Thèse, Wageningen, citée par Auriol et JARrige.

Poly J. et Vissac B:; I959. Interprétation des résultats de contrôle laitier en vue du testage des taureaux d'insémination. Bull. Techn. Inf. Ing. Serv. Agric., 145, 841-862.

Poutous M., I 963 . Résultats préliminaires sur le testage des taureaux d'après la production en matière azotée de leurs filles. Indust. lait., 203, $25^{8-260 .}$

RoBertson A., Waite R., and White J. C. 1956. Variations in the chemical composition of milk with particular reference to the solids-not-fat. II. The effect of Heredity. J. Dairy Res., 23, 82-9I.

Robertson A., 196r. Reviews of the progress of dairy science. Section A. Physiology. Genetics of Dairy cattle. J. Dairy Res., 28, I95-207.

Tyler W. J., I958. Genetic aspects of solids-non-fat and its components. J. Dairy Sci., 41, 447-449.

Vissac B., I $96 \mathbf{I}$. Principes d'analyse et de décomposition des variances et covariances, pour des classifications hiérarchiques non orthogonales. (Non publié).

Von Krosigk C. M., Young J. O. and Richardson J. A., ig6o. Genetic. Influences on the composition of cow's milk. J. Dairy Sci., 43, 877 . 